\title{
Venturino: análise da variação de pressão em um tubo de Venturi utilizando Arduino e sensor de pressão
}

Venturino: analysis of pressure variation in a Venturi's tube using Arduino and pressure sensor

\author{
A. S. Cid*1@, T. Correa \\ ${ }^{1}$ Centro Federal de Educação Tecnológica Celso Suckow da Fonseca, Valença, RJ, Brasil \\ ${ }^{2}$ Universidade do Estado do Rio de Janeiro, Instituto de Aplicação Fernando Rodrigues da Silveira, Departamento de Ciências \\ da Natureza, Rio de Janeiro, RJ, Brasil
}

Recebido em 30 de Novembro, 2018. Aceito em 07 de Janeiro, 2019.

\begin{abstract}
Geralmente os laboratórios didáticos das escolas de ensino médio e das instituições de ensino que ofertam graduações em Física e áreas correlatas não possuem equipamentos e/ou práticas experimentais sobre hidrodinâmica. Aplicações de relevância tecnológica tais como o tubo de Pitot ou o tubo de Venturi são estudados, geralmente, como simples aplicações matemáticas da Equação de Bernoulli para fluidos ideais. Visando contribuir para a remediação deste problema é proposto nesse artigo a construção de um tubo de Venturi fabricado com materiais de baixo custo e equipado com uma placa microcontroladora Arduino e sensores de pressão modelo BMP280. O aparato proposto nesse artigo permite a visualização de gráficos, gerados em tempo real, da variação da pressão interna em um ponto com o transcorrer do tempo e estabelece relações empíricas entre pressão, velocidade e área do tubo. O aparato já foi utilizado em cursos técnicos integrados do CEFET/Valença e em curso de Física Experimental que compõe a grade curricular da graduação em Engenharia de Alimentos da mesma instituição. Essa prática faz parte de um projeto que visa criar recursos didáticos para desenvolver atividades de ensino baseados no conceito de aprendizagem ativa, utilizando o método Predizer - Observar - Explicar (POE).
\end{abstract}

Palavras-chave: Hidrodinâmica, Arduino, Tubo de Venturi, laboratório didático.

Usually, didactic labors inside schools and inside institutions which offers Physics graduations or related areas does not have equipments or experimental practice about hydrodynamics theme. Important technological applies like Pitot or Venturi tubes are considered only from mathematics perspectives without connection between industry or technological fields and the developed theory. This paper proposes a Venturi tube construction using low cost materials to solve at least in part the lack of hydrodynamics experimental practices. The tube is connected to model BMP280 pressure sensors controlled by a microcontroller board arduino. The apparatus allows to print graphics, created in real time, about how pressure changes at a fixed point over time and establish empirics links between pressure, fluid speed and tube area. It had done in technical courses from CEFET/Valença and in an experimental Physics course which composes the Food Engineering grade. This experiment is inside a project that aims to create didactic resources to develop teaching activities supported by learning activities concepts through Predict - Observe - Explain method (POE).

Keywords: Hydrodinamics, Arduino, Venturi's Tube, didatic laboratory.

\section{Introdução}

A elaboração de atividades experimentais que visam compreender o funcionamento dos tubos de Venturi e de Pitot permitem desenvolver, simultaneamente, conceitos básicos da Mecânica dos Fluidos e estabelecer conexões com o universo científico, tecnológico e social. A Mecânica dos Fluidos possui diversas aplicações interessantes, por exemplo, na aviação. Para endossar a relevância do tema basta destacar que o tubo de Pitot é um dispositivo utilizado em aviões capaz de gerar dados sobre a velocidade de navegação e fundamental para garantir a estabilidade e segurança dos voos. Inclusive, a perícia que investigou as causas do acidente protagonizado pelo Air Bus 447 em
2009, aeronave da Air France, na rota Rio de Janeiro Paris, apontou as falhas do sistema de descongelamento como causa principal para a queda da aeronave [1]. Apesar da relevância do conteúdo, nos cursos de ensino médio e graduações, normalmente, não são estabelecidas conexões entre a teoria e suas aplicações mais relevantes. Ao examinar os principais livros didáticos adotados, tanto do ensino médio [2-7] quanto do ensino superior [8-11], nota-se que quando o tema é desenvolvido, quase sempre é seguida a mesma sequência didática: definição de fluxo, equação da continuidade, equação de Bernoulli e dois problemas tradicionais - o tubo de Venturi e o tubo de Pitot. Entretanto, frequentemente, ambos são abordados como meros exemplos focados em manipulações mate-

*Endereço de correspondência: asilvacid@gmail.com 
máticas da equação de Bernoulli, descontextualizando-os das aplicações.

À metodologia tradicional, focada em aplicações matemáticas, soma-se o fato dos laboratórios didáticos das escolas e universidades estarem desprovidos, salvo algumas exceções, de equipamentos que permitam realizar práticas experimentais adequadas e relevantes de Mecânica dos Fluidos. Ainda, quando os equipamentos estão disponíveis e práticas experimentais são realizadas, comumente, estão construídas para servirem de simples ilustração teórica baseadas no modelo de laboratório estruturado (cookbook, em inglês). Nesse modelo o estudante recebe o equipamento e um roteiro para montar e/ou realizar os experimentos que os levam a respostas formatadas a fim de serem comparadas com o resultado teórico esperado sem que aconteçam elaboração de hipóteses, construção de modelos e discussões sobre suas limitações de aplicabilidade.

Em vista de sanar, ao menos em parte, a escassez de atividades experimentais sobre hidrodinâmica no contexto discutido, relatamos no presente artigo a construção de um tubo de Venturi fabricado com materiais de baixo custo associado a uma placa microcontroladora Arduino e sensores de pressão, denominado a partir desse instante como Venturino. O microcontrolador Arduino foi criado em 2005, desenvolvido com a finalidade de ser uma solução de baixo custo para estudo de programação, automação e designs em geral. Desde então tem sido empregado com sucesso em propostas que associam o ensino de ciências, em especial da Física, com TIC's (Tecnologias de Informação e Comunicação). Na literatura podemos encontrar diversas propostas para sua utilização no ensino de Física [12-15] contudo é inédita a associação do Arduino ao tubo de Venturi para aferir a pressão hidrodinâmica em tempo real a fim de levar o estudante a realizar a conexão entre os conceitos básicos que sustentam a equação de Bernoulli: pressão do fluido, área da seção transversal do tubo e velocidade de escoamento do fluido. Além disso, ainda é possível encontrar trabalhos que propõem experimentos de hidrodinâmica construídos com materiais de baixo custo [16-23], mas também é inédita a construção de um tubo de Venturi que associa a tecnologia Arduino e materiais de baixo custo. O equipamento proposto é portátil, leve e gerou um custo de aproximadamente 80 reais.

$\mathrm{O}$ aparato proposto neste artigo foi utilizado em duas turmas do $2^{\circ}$ ano do ensino médio integrado do $\mathrm{CE}$ FET/Valença e em uma turma do curso de Física Experimental que compõe a grade curricular do curso de Engenharia de Alimentos do CEFET/Valença.

Ao final desse trabalho é apresentada, ainda, uma sugestão de construção de atividade baseado no método Predizer - Observar - Explicar (POE) em contraponto ao modelo de laboratório estruturado. O POE foi originalmente concebido como uma ferramenta de avaliação formativa da aprendizagem para ser empregado concomitantemente a um experimento qualitativo, demonstrado pelo professor em aulas teóricas, após ministrar o conteúdo. Porém atualmente, a metodologia POE tem sido mais utilizada como uma estratégia de promoção de aprendizagem em diversas áreas de ciências [24-26] e já existem pesquisas que comprovam a eficiência do método também com simulações computacionais [27-28] e vídeos [29]. O POE hoje está presente em diversos países, desde a educação básica até os primeiros anos do ensino superior, e se insere na tendência mundial conhecida como metodologias de aprendizagem centrada no estudante, uma vez que torna o aluno autônomo e sujeito da construção do próprio conhecimento [30].

O trabalho segue então a seguinte estrutura: na segunda seção é feito um resumo teórico do tema a ser explorado; na terceira seção é descrita a montagem do arranjo experimental, materiais necessários, programação e custo estimado; na quarta seção são apresentadas sugestões de atividades experimentais realizadas com o Venturino; na quinta seção são apresentados resultados obtidos com o uso do aparato e da sua aplicação em aulas experimentais; por fim, a sexta seção encerra o trabalho com as conclusões.

\section{A Equação de Bernoulli e o Tubo de Venturi}

A Equação de Bernoulli, ou Princípio de Bernoulli, foi apresentada na obra Hydrodynamica (1738) pelo matemático suíço Daniel Bernoulli [31]. A equação representa uma aplicação do princípio de conservação de energia para fluidos ideais, que nos possibilita relacionar a velocidade do fluido com a pressão no mesmo. Inspirado na equação de Bernoulli, o físico italiano Giovanni Battista Venturi criou o tubo de Venturi, um aparato utilizado para obter a velocidade do escoamento de um líquido incompressível. No tubo um líquido atravessa uma região com maior seção transversal e em seguida outra região de menor seção transversal. A partir da diferença de pressão entre as duas regiões, aferida através do deslocamento de colunas do líquido, é possível obter as velocidades do líquido nas regiões. Um exemplo de tubo de Venturi pode ser visto na Figura 1

Considerando o tubo da Figura 1, de acordo com a equação de Bernoulli, temos a relação entre os pontos 1

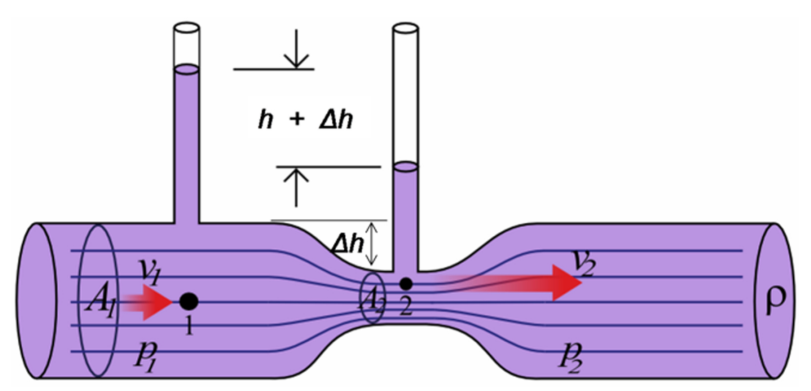

Figura 1: Tubo de Venturi 
e 2:

$$
p_{1}+d g h_{1}+\frac{d v_{1}^{2}}{2}=p_{2}+d g h_{2}+\frac{d v_{2}^{2}}{2}
$$

Onde $\mathrm{p}_{\mathrm{n}}$ é a pressão no ponto $\mathrm{n}$, d é a densidade do fluido, g é a aceleração da gravidade, $\mathrm{h}_{\mathrm{n}}$ é a altura do ponto $\mathrm{n}$ em relação ao chão e $\mathrm{v}_{\mathrm{n}}$ a velocidade do fluido no ponto $n$. De acordo com a equação da continuidade para fluidos incompressíveis, temos que,

$$
A_{1} v_{1}=A_{2} v_{2} \Rightarrow v_{2}=\frac{A_{1} v_{1}}{A_{2}}
$$

Onde $\mathrm{A}_{\mathrm{n}}$ é a área de seção reta do tubo em n. Inserindo a Eq. 2 na Eq. 1 e isolando $v_{1}$, teremos:

$$
v_{1}=\sqrt{\frac{\left(\frac{2}{d}\left(p_{2}-p_{1}\right)+2 g\left(h_{2}-h_{1}\right)\right)}{1-A_{1}^{2} / A_{2}^{2}}}
$$

Portanto, é possível obter as velocidades $\mathrm{v}_{\mathrm{n}}$ através de medidas experimentais das pressões $\mathrm{p}_{1}$ e $\mathrm{p}_{2}$, das alturas $\mathrm{h}_{1}$ e $\mathrm{h}_{2}$ e das áreas $\mathrm{A}_{1}$ e $\mathrm{A}_{2}$.

\section{Arranjo Experimental}

O arranjo experimental consiste do Venturino (Figura 2), um aspirador de pó para gerar fluxo de ar no interior do tubo e um computador para visualização dos gráficos de pressão interna em função do tempo, gerados pelo software Arduino IDE (versão 1.8.5) [32]. O aspirador utilizado foi o modelo Easy Clean Turbo da Philco de $1800 \mathrm{~W}$ de potência elétrica. O custo aproximado do Venturino (não incluso aspirador e computador) é de 8 reais. Apresentaremos nas seções seguintes os materiais utilizados e descreveremos o processo de montagem.

\subsection{O tubo}

Para a confecção do tudo foram utilizados os seguintes materiais:

- Um tubo de PVC de $75 \mathrm{~mm}$ de diâmetro e comprimento de $50 \mathrm{~cm}$;

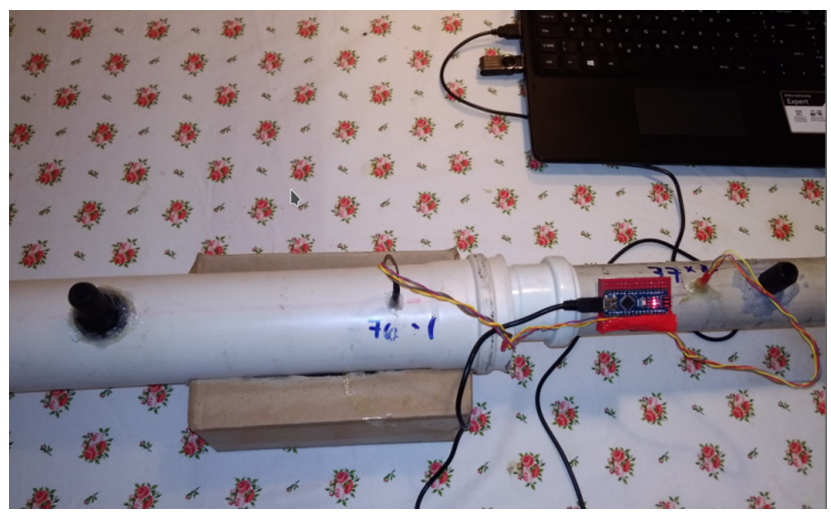

Figura 2: Venturino
- Um tubo de PVC de $50 \mathrm{~mm}$ de diâmetro e comprimento de $34 \mathrm{~cm}$;

- Uma luva de redução PVC soldável de 75 para 50 $\mathrm{mm}$;

- Dois bicos para torneira de plástico com diâmetro de $3 / 4$;

- Furadeira, broca e serra copo;

- Massa adesiva (durepoxi) e adesivo plástico para PVC.

A construção do tubo é simples, consiste no acoplamento dos dois tubos (de 75 e $50 \mathrm{~mm}$ ) através da luva de redução soldável e da aplicação do adesivo plástico para PVC. Ainda, em cada tubo, aproximadamente na metade do comprimento, é feita uma abertura com uma serra copo para instalação do bico para torneira. O bico, após encaixado na abertura, é fixado com a massa adesiva. Finalizada a construção do tubo devemos acoplar a saída do aspirador de pó a uma das extremidades do tubo (a extremidade utilizada não influencia nos resultados obtidos).

\subsection{Arduino e sensor de pressão}

\section{Materiais e montagem}

Para realizar a aferição da pressão em tempo real no tubo de Venturi são necessários os seguintes componentes:

- Uma placa Arduino;

- Dois sensores de pressão BMP280;

- 9 jumpers macho/fêmea;

- Uma protoboard (opcional);

- Cola de silicone.

O sensor de pressão BMP280 é um sensor piezo-resistivo, capaz de converter forças atuantes sobre ele em diferença de potencial elétrico. No raio de funcionamento entre 940 e $1100 \mathrm{hPa}$, à temperatura ambiente, apresenta uma exatidão de aproximadamente $\pm 0.12 \mathrm{hPa}$ (equivalente a cerca de $\pm 1 \mathrm{~m}$ ) [33].

Em nosso aparato utilizamos a placa Arduino NANO, no entanto outras placas podem também ser utilizadas, como a mais conhecida Arduino UNO. A protoboard é opcional, facilita as ligações do circuito, no entanto não é essencial à construção do aparato. A instalação dos dois sensores de pressão BMP280 foi realizada fazendo um furo em cada tubo, de espessura suficiente para permitir a passagem dos quatro fios necessários à instalação de cada um. Em seguida os sensores foram puxados para dentro do tubo utilizando um arame como guia, preso na extremidade dos fios. Finalmente, os furos pelos quais passam os fios são vedados com cola de silicone. A cola também permite manter os sensores fixos. A Figura 3 exibe o detalhe do sensor posicionado dentro do tubo.

\section{Circuito}

A ligação do sensor ao Arduino não envolve muitas complexidades. O sensor possui seis conexões, conforme é visto na Figura 4 das quais apenas quatro serão utilizadas em ambos os sensores: VCC, GND, SCK/SCL e SDA/SDI. 


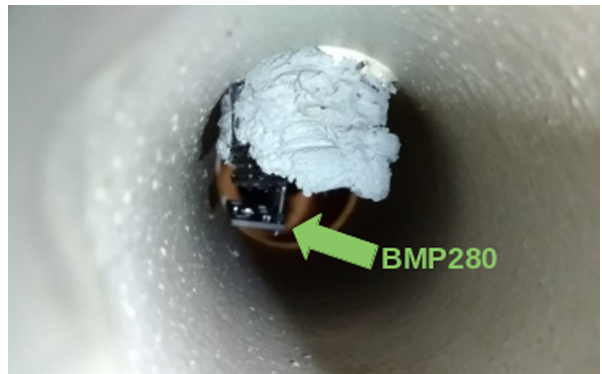

Figura 3: Detalhe do sensor de pressão instalado dentro do tubo.

Os sensores comunicam-se com o Arduino através de "endereços" pré-estabelecidos, sendo o mesmo para todos os modelos de BMP280, no entanto, devido ao uso simultâneo de dois sensores torna-se imperioso a alteração do "endereço" de um deles para que não haja conflito. Essa operação é feita ao conectar a entrada SDO de um dos sensores ao pino $5 \mathrm{~V}$ do Arduino. As conexões VCC e GND de cada sensor são conectadas, respectivamente, às entradas $5 \mathrm{~V}$ e GND do Arduino, enquanto as outras conexões devem ser conectadas em pinos apropriados, que podem ser distintos em cada modelo de placa. Especificamente para a placa Arduino NANO (e também para a UNO) os pinos apropriados são os pinos A4 (SDA/SDI) e A5 (SCL/SCK). Como alguns pinos do Arduino receberão múltiplas conexões, a utilização da protoboard é recomendada. Vale ressaltar que o sensor pode apresentar os pinos soldados ou não e isso depende do local de aquisição. Caso os pinos não sejam soldados será necessário, ainda, um ferro de solda. O esquema do circuito pode ser visto na Figura 5.

\section{Código}

Com todo o aparato instalado e sensores conectados à placa Arduino, resta efetuar o envio do código para a
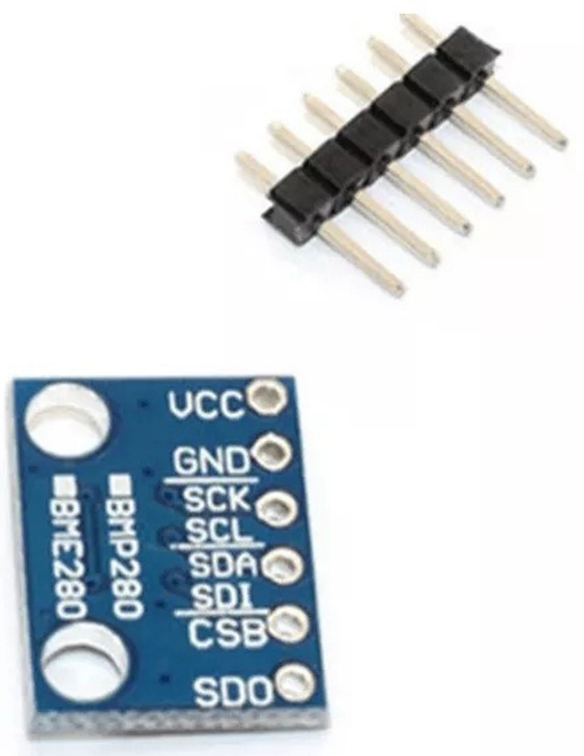

Figura 4: Sensor de pressão BMP280.

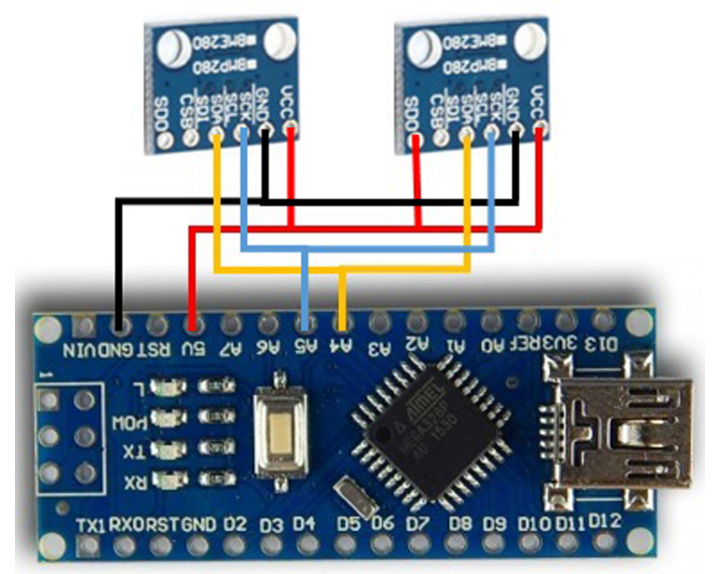

Figura 5: Esquema do circuito.

mesma. Para isso é preciso acessar a página do Arduino [16] e baixar o programa Arduino IDE, disponível gratuitamente para Windows, Mac OS e Linux. Após baixado e instalado, deve-se adicionar na pasta do programa, em libraries, as bibliotecas necessárias, que podem ser encontradas em nosso github [34], assim como o código. Adicionadas as bibliotecas, basta abrir o arquivo do código e carregar para a placa, previamente conectada à porta USB do computador. Em seguida, na Arduino IDE, clique em Ferramentas e em Plotter Serial, para observar o gráfico das pressões em função do tempo. Inicialmente, com o aspirador desligado, os gráficos das pressões devem estar próximos, no entanto, devido aos sensores não serem perfeitamente iguais, pode haver uma pequena diferença nos valores da pressão, possível de ser corrigida através de modificação no código, em local indicado no mesmo através de comentários. Feita a correção o código deve ser novamente carregado para a placa. Um passo a passo da utilização da Arduino IDE pode ser encontrada na seguinte referência [35]. O código, além de poder ser baixado em nosso github, encontra-se no Apêndice do artigo. Na Figura 6 podemos ver o gráfico visualizado na serial plotter, inicialmente com o aspirador desligado e em seguida com ele ligado. $\mathrm{O}$ eixo vertical apresenta a pressão nos tubos em Pascal, enquanto o eixo horizontal apresenta o tempo transcorrido desde o momento em que o plotter serial foi aberto, em milissegundos. No aparato a curva azul corresponde ao sensor de pressão instalado no tubo maior $(75 \mathrm{~mm})$, enquanto a curva vermelha corresponde ao sensor de pressão instalado no tubo menor $(50 \mathrm{~mm})$.

\section{Experimentos}

Essa seção objetiva sugerir e discutir experimentos possíveis de serem realizados com o Venturino. 


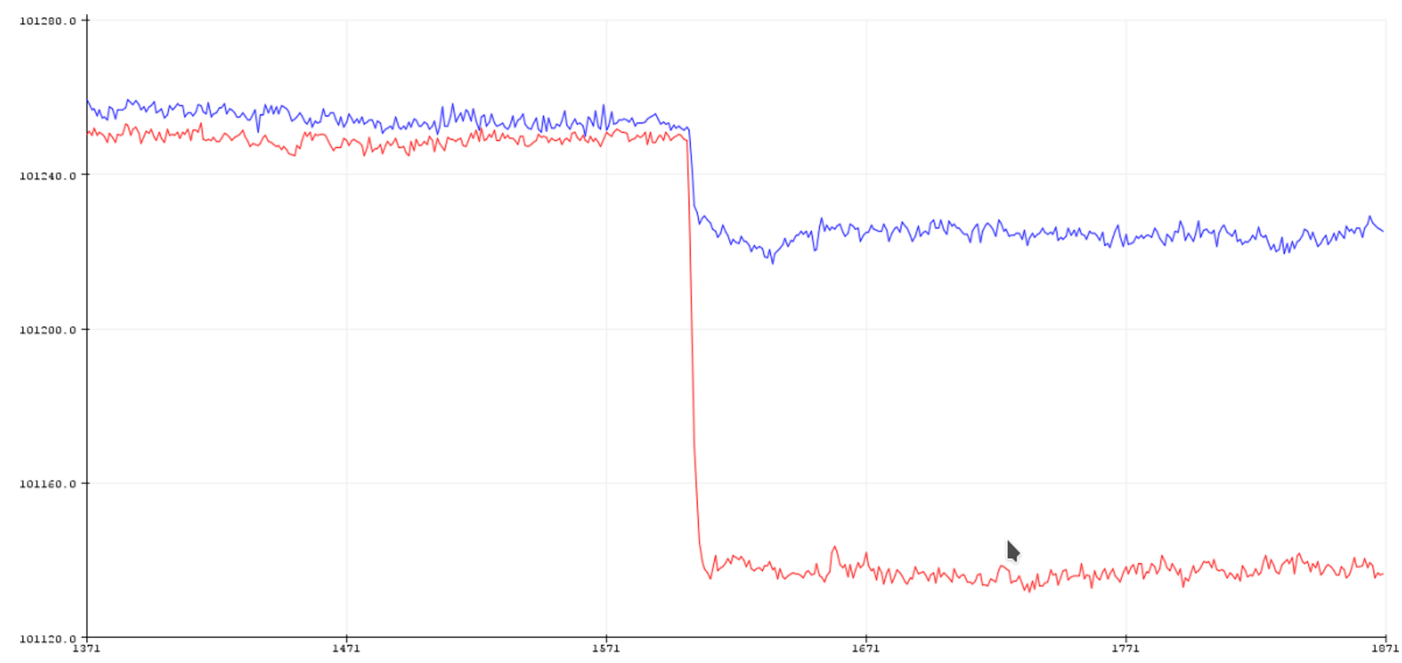

Figura 6: Gráfico visualizado na serial plotter em Niterói a 25 m acima do nível do mar.

\subsection{Experimento 1: Monitoramento da pressão interna do tubo}

O Venturino permite correlacionar, através de gráficos construídos em tempo real, a pressão interna do fluido e a área da seção transversal do tubo pelo qual desloca-se esse mesmo fluido. Um fluxo de ar é gerado quando o aspirador de pó é ligado e a pressão é monitorada ao longo do tempo nos pontos onde estão localizados os sensores BMP280. De acordo com a Figura 6, a pressão interna do fluido é maior na região monitorada pelo sensor correspondente a curva azul do que a pressão na região monitorada pelo sensor correspondente a curva vermelha. Essa observação já estabelece uma relação empírica entre área e pressão, além de permitir o entendimento da Eq. 1 com maior profundidade. Esse experimento permite concluir que quanto menor a área de seção transversal menor é a pressão interna do fluido.

\subsection{Experimento 2: Efeito da pressão interna sobre bolinhas de isopor}

Um segundo experimento interessante consiste em tapar os dois bicos de saída de ar do Venturino com bolinhas de isopor (Figura 7) e verificar que a bolinha associada ao diâmetro maior (bico 1) cai antes da bolinha associada ao diâmetro menor (bico 2) ao realizar rotação em torno do eixo transversal. Para entender o efeito é necessário considerar que as bolinhas estão submetidas, simultaneamente, a pressão interna do fluido e a pressão atmosférica externa. A pressão atmosférica é igual sobre ambas as bolinhas, enquanto a pressão interna é menor sobre a bolinha posicionada no bico 2 conforme discutido no Experimento 1. A força resultante sobre as duas bolinhas aponta no sentido de fora para dentro do tubo, uma vez que a pressão atmosférica é maior do que a pressão ao longo do tubo quando existe o fluxo de ar (Figura 6). Contudo, no bico 1 a diferença entre a pressão atmosférica e a pressão interna é menor do que a diferença entre a pressão atmosférica e a pressão interna

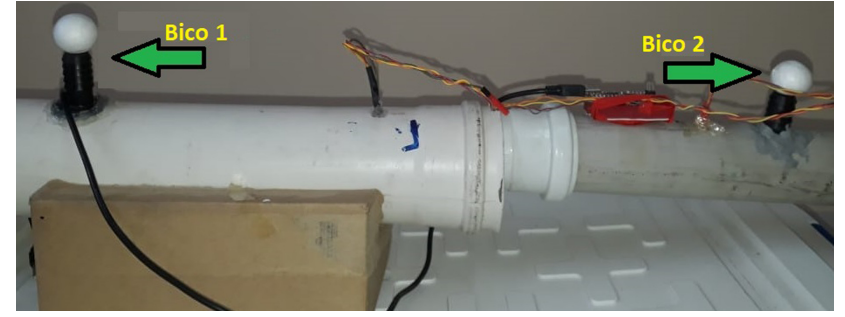

Figura 7: Demonstração do Experimento 2.

no bico 2. Através desse raciocínio explica-se o fato da bolinha 2 cair posteriormente a bolinha 1 quando o tubo é rotacionado lentamente. Esse experimento é de fácil realização e serve para mostrar que a queda das bolinhas não depende somente da pressão interna do fluido e sim da resultante das forças que atuam sobre as mesmas. Ressalta-se ainda que o efeito é o mesmo independente da saída do tubo pela qual o aspirador é acoplado e a Física do problema é análoga se ao invés do aspirador for utilizado um equipamento que injete ar no interior do tubo.

\subsection{Experimento 3: Efeito das saídas de ar nas medidas de pressão.}

A equação de Bernoulli, sabemos, é obtida através da lei de conservação de energia e, consequentemente, não é aplicável em situações para as quais existem fontes ou saídas de ar ao longo do tubo. No Venturino existem duas saídas de ar que podem ser utilizadas para suscitar discussões sobre a validade da equação 1. Essa discussão torna-se possível ao realizar uma comparação entre a velocidade teórica, calculada através da equação 3, e a velocidade experimental. A velocidade experimental pode ser estimada conhecendo o fluxo de ar associado ao modelo de aspirador empregado. Essa informação, algumas vezes, é possível obter no manual do equipamento. Quando o fluxo não constar no manual é possível ter 
uma ideia de ordem de grandeza através da consulta a manuais de outros modelos semelhantes.

\section{Resultados}

Nesta seção são apresentados os resultados vinculados aos testes iniciais, além dos resultados obtidos na aplicação dos experimentos propostos na seção anterior.

\subsection{Teste de validação do sensor BMP280}

O Venturino foi submetido a testes preliminares entre os dias seis e vinte de abril de dois mil e dezoito em duas cidades fluminenses: Niterói e Valença. O primeiro teste consistiu em comparar a diferença de pressão atmosférica entre as duas cidades, aferidas com o aparato, e a diferença de pressão calculada utilizando o princípio de Stevin e altitudes das cidades provenientes de uma base de dados [36]. Em Niterói o valor médio obtido para a pressão atmosférica foi de $101240 \mathrm{~Pa}$, enquanto em Valença o valor médio obtido foi igual a $95595 \mathrm{~Pa}$, resultando numa diferença de pressão de $5645 \mathrm{~Pa}$ entre as duas cidades. Através de consulta à base de dados Daft Logic obtivemos para a cidade de Niterói uma altitude de $25 \mathrm{~m}$ acima do nível do mar, e para a cidade de Valença uma altitude de $535 \mathrm{~m}$ acima do nível do mar. Utilizando o princípio de Stevin e considerando entre as duas localidades densidade do ar constante igual a $1,165 \mathrm{~kg} / \mathrm{m}^{3}$, e aceleração da gravidade constante igual a $9,788 \mathrm{~m} / \mathrm{s}^{2}[37]$, obtivemos uma diferença de pressão igual a $5815,5 \mathrm{~Pa}$ entre as duas cidades. Podemos observar que a diferença de pressão obtida com o Venturino e a calculada diferem aproximadamente em $3 \%$, sendo a primeira menor, justamente devido à densidade do ar e a aceleração da gravidade diminuírem com a altitude.

\subsection{Cálculo das velocidades do fluido em cada seção do Venturino}

Um segundo teste consiste em comparar as velocidades obtidas através das Eqs. 2 e 3 com as velocidades tabeladas em manuais de aspiradores de pó. O experimento foi realizado com o aspirador ligado na cidade de Valença, obtendo o gráfico apresentado na Figura 8. As curvas azul e vermelha apresentam, respectivamente, as pressões nos tubos de 75 e $50 \mathrm{~mm}$. Utilizando as Eqs. 3 e 2, obtémse as respectivas velocidades do ar nos tubos maior e menor: 8,08 m/s e 18,11 m/s. O manual do modelo de aspirador utilizado não faz referência à velocidade do fluxo de ar, no entanto o valor obtido para a mesma no tubo de menor diâmetro (que possui mesmo diâmetro da saída do aspirador), é compatível com velocidades associadas ao fluxo gerado por aspiradores de outros modelos semelhantes e com mesma potência Ao comparar as velocidades calculadas com aquelas fornecidas nos manuais de aspiradores pode permitir, ainda, uma discussão sobre a validade da equação de Bernoulli e, portanto de conservação de energia.

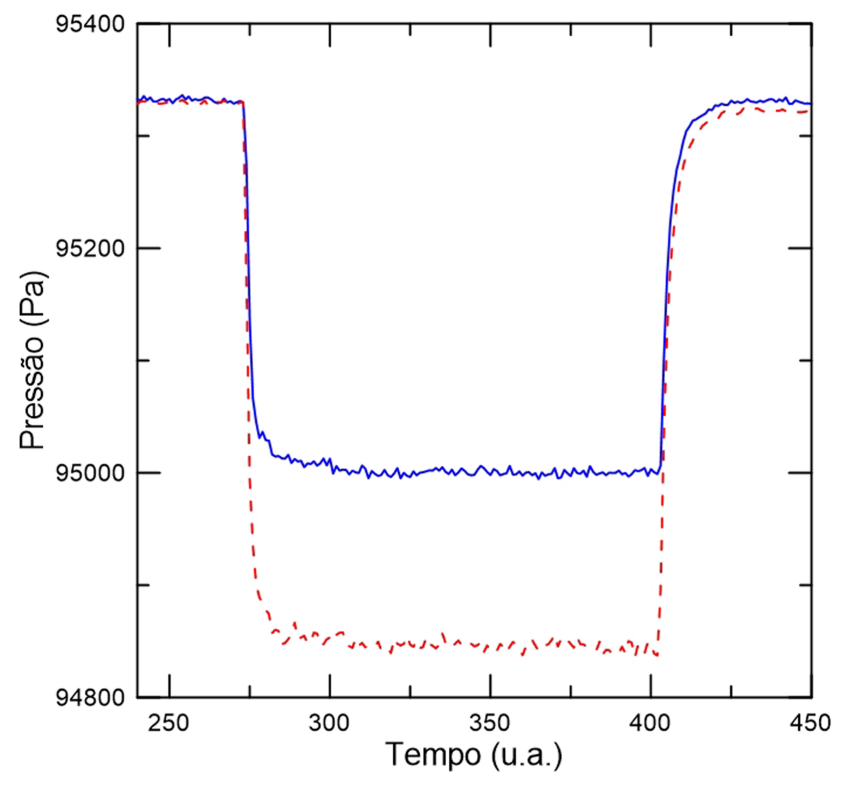

Figura 8: Gráfico obtido na cidade de Valença. A curva azul representa a pressão no tubo de $75 \mathrm{~mm}$, já a curva vermelha a pressão no tubo de $50 \mathrm{~mm}$.

\subsection{Aplicação dos experimentos sugeridos}

Os experimentos propostos nesse artigo foram reproduzidos em sala de aula dentro da perspectiva do método de aprendizagem ativa conhecido como Predizer-ObservarExplicar (POE) [38]. Não constitui objetivo desse trabalho discutir a metodologia de ensino-aprendizagem empregada mas sim subsidiar escolas e professores com experimentos de baixo custo no campo da hidrodinâmica. Contudo, para que o leitor sacie a necessidade de sugestão para aplicação dos experimentos de acordo com um referencial teórico indica-se, veementemente, a leitura do trabalho "Uma proposta de ensino do princípio de Stevin através do método Predizer-Observar-Explicar (POE)"[30]. As considerações sobre metodologia para a construção das aulas de hidrodinâmica para as quais foram utilizadas o Venturino faz-se análoga a discussão do trabalho citado. No apêndice o leitor poderá consultar a atividade POE, aplicada nas salas de aula, como sugestão de utilização dos experimentos. Ressalta-se que a atividade POE sugerida no apêndice gerou resultados positivos no sentido de que grande parte dos alunos construíram qualitativamente as relações entre pressão, velocidade e diâmetro do tubo necessárias para o entendimento da equação de continuidade e da equação de Bernoulli para fluidos ideais. A confirmação do sucesso está amparada pela análise prévia das respostas dos alunos para a atividade e de outros instrumentos avaliativos que foram aplicados posteriormente. Todavia a discussão metodológica, os recursos didáticos empregados, os conflitos cognitivos emergentes nas respostas geradas pelos alunos e a análise dos instrumentos de avaliação são o escopo de um segundo artigo. 


\section{Conclusões}

O Venturino foi construído e aplicado seguindo os experimentos sugeridos na terceira e quarta seção. A aplicação foi realizada em duas turmas de $2^{\circ}$ ano dos cursos de Alimentos e Química e em uma turma do curso de graduação em Engenharia de Alimentos, ambas do CEFET/Valença. Em cada um dos casos os experimentos foram empregados com sucesso aliados a metodologia POE de aprendizagem ativa. Os experimentos realizados permitiram discutir a variação da pressão com a altitude através dos gráficos obtidos em Niterói e em Valença, além de estabelecer uma relação empírica entre a pressão interna de um fluido e a área da seção transversal do tubo que o contém através dos gráficos gerados pelos sensores BMP280 controlados pela plataforma Arduino. Finalmente, a análise dos dados de pressão gerados pelos sensores permite obter as velocidades do ar no interior de cada tubo. Para despertar o interesse pela compreensão de hidrodinâmica por parte dos alunos foi feita a leitura de uma reportagem sobre acidentes aéreos ocasionados por problemas com o tubo de Pitot [1]. A contextualização do conteúdo aliada aos recursos experimentais, motivaram maior participação dos alunos nas aulas de Física posteriores e aguçaram a curiosidade. Portanto, esse trabalho fornece subsídio para desenvolver o tema hidrodinâmica em sala de aula através da construção de um aparato de baixo custo e realiza propostas de atividades lúdicas e experimentais. Essas atividades permitem construir as relações entre as grandezas relevantes para o entendimento inicial do tema hidrodinâmica: velocidade, pressão e área da seção transversal.

\section{Material suplementar}

O seguinte material suplementar está disponível online: Apêndice.

\section{Referências}

[1] https://brasil.estadao.com.br/noticias/geral, justica-francesa-reconhece-crime-na-queda-dovoo-447,616651, acessado em 12/06/2018.

[2] C. Xavier e B. Barreto, Fúsica aula por aula (FTD, São Paulo, 2010), v. 2, p. 80.

[3] L.A. Guimarães e M.F. Boa, Mecânica (Galera, Niterói, 2010), v. 1, p. 420.

[4] A.G. Filho e C. Toscano, Física: Interação e Tecnologia (Leya, Lisboa, 2016), $2^{\underline{a}}$ ed.

[5] L.F. Fuke e K. Yamamoto, Fúsica para o ensino médio (Saraiva, São Paulo, 2016), $4^{\mathrm{a}} \mathrm{ed}$.

[6] A. Gaspar, Compreendendo a Fúsica (Ática, São Paulo, 2016), $3^{\underline{a}}$ ed.

[7] A. Máximo, B. Alvarenga e C. Guimarães, Fúsica: Contexto e Aplicações (Scipione, Rio de Janeiro, 2016), 2ª ${ }^{\mathrm{a}} \mathrm{ed}$.

[8] D. Halliday, R. Resnick e J. Walker, Fundamentos de Física (LTC, Rio de Janeiro, 2016), v. 2, p. 85.

[9] D.K. Randall, Fúsica: Uma abordagem estratégica (Bookman, Porto Alegre, 2009), v. 2, p. 465.
[10] H.D. Young e R.A. Freedman, Fúsica 2: Termodinâmica e Ondas (Addison Wesley, São Paulo, 2003), v. 2, p. 85.

[11] H.M. Nussenzveig, Curso de Física Básica (Blucher, São Paulo, 2014), v. 2, p. 25.

[12] M.A. Cavalcante, C.R.C. Tavolaro e E. Molisani, Revista Brasileira de Ensino de Física 33, 4503 (2011).

[13] H. Cordova e A.C. Tort, Revista Brasileira de Ensino de Física 38, 2308 (2016).

[14] A.R. Souza, A.C. Paixão, D.D. Uzêda, M.A. Dias, S. Duarte e H.S. Amorim, Revista Brasileira de Ensino de Física 33, 1702 (2011).

[15] L.R.M. Carvalho e H.S. Amorim, Revista Brasileira de Ensino de Física 36, 3501 (2014).

[16] L.H. Barbosa, C.E. Mora, P.H. Talero e J.O. Organista, Revista Brasileira de Ensino de Física 33, 4309 (2011).

[17] V.L.B. Jesus e M.A.V. Macedo Junior, Revista Brasileira de Ensino de Física 33, 1507 (2011).

[18] L. Verchenko, A.G. Dickman e J.R.F. Ferreira, Revista Brasileira de Ensino de Física 31, 3301 (2009).

[19] B.C.S. Grandi, Caderno Brasileiro de Ensino de Física 7, 73 (1990).

[20] E.J. Souza, Caderno Brasileiro de Ensino de Física 34, 530 (2017).

[21] A.A.D. Filho, Caderno Brasileiro de Ensino de Física 13, 76 (1996).

[22] R. Qin e C. Duan, Jounal of Physics: Conf. Ser. 916, 012038 (2017).

[23] J. Haysom e M. Bowen, Predict, Observe, Explain: Activities Enhancing Scientific Understandin (NSTA Press, Arlington, 2010).

[24] C.W. Liew Effectiveness of Predict-Observe-Explain Technique (Lambert Academic Publishing, Düsseldorf, 2009)

[25] R.J. Santos e D.G.G. Sasaki Revista Brasileira de Ensino de Física 37, 3506 (2015).

[26] D.G.G. Sasaki e V.L.B. Jesus Revista Brasileira de Ensino de Física 39, 2403 (2017).

[27] P.K. Tao e R.F. Gunstone Journal of Research in Science Teaching 36859 (1999).

[28] O. Balen e P.A. Netz Acta Scientiae 7, 29 (2005).

[29] M. Kearney e D.F Treagust Australian Journal of Educational Technology 1764 (2001).

[30] A.S. Cid e D.G.G. Sasaki, in Anais do XVII Encontro de Pesquisa em Ensino de Física, (SBF, Campos do Jordão, 2018).

[31] J. Westerweel, Nederlands Tijdschrift voor Natuurkunde 82, 218 (2016).

[32] https://www.arduino.cc/en/Main/Software, acessado em 31/05/2018.

[33] Bosch Sensortec, Data Sheet: BMP 280 Digital Pressure Sensor (Bosch, Reutlingen, 2015).

[34] https://github.com/thiagoca3/venturino, acessado em 06/08/2018.

[35] T. Correa, Arduino \& Pi Lab, disponível em https://arduinopilab.wordpress.com/2017/03/28/ arduino-primeiros-passos/, acessado em 31/05/2018.

[36] https://www.daftlogic.com/sandbox-google-mapsfind-altitude.htm, acessado em 31/05/2018.

[37] M.A. Sousa e A.A. Santos, Rev. Bras. Geof. 28, 2 (2010).

[38] R.T. White e R.F. Gunstone, Probing Understanding (London Falmer, 1992). 